\section{Benzodiazepines, antidepressants and addiction: A plea for conceptual rigor and consistency}

\author{
Richard Balon ${ }^{1,2,3^{*}}(\mathbb{D})$, Edward K Silberman $4^{*}$, Vladan Starcevic ${ }^{5^{*}}(\mathbb{D})$, \\ Fiammetta Cosci ${ }^{6,7}$, Rafael C Freire 8,9 (D), Antonio E Nardi 9 (D), \\ Karl Rickels ${ }^{10}$ and Richard Shader ${ }^{11}$
}

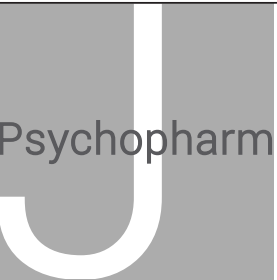

Journal of Psychopharmacology $1-4$ (c) The Author(s) 2019 Article reuse guidelines: sagepub.com/journals-permissions DOI: $10.1177 / 0269881119878171$ journals.sagepub.com/home/jop

(A) SAGE
The editorial by Jauhar et al. (2019) discusses the recently published concerns about "addiction" to antidepressants (mainly serotonin reuptake inhibitors and serotonin and noradrenaline reuptake inhibitors) in light of an increasing number of publications addressing antidepressant withdrawal symptoms. They (Jauhar et al., 2019) ask a crucial question ("Are antidepressants addictive?"), examine conceptual and methodological issues and arrive at a conclusion that "there is minimal evidence, using established classification systems and concepts, that antidepressants should be classified as addictive substances" (p.657). We agree with their conclusion that antidepressants are not addictive and that the main argument invoked in support of addiction to antidepressants - the presence of withdrawal symptoms - is not valid. However, we would like to point that the same standard used for antidepressants in this regard should also be applied to other pharmacological agents - in particular to benzodiazepines. We are concerned that benzodiazepines (positive allosteric modulators of gamma aminobutyric acid [GABA-A] receptor, benzodiazepine site) are frequently labelled as "addictive" and are included in the Diagnostic and Statistical Manual of Mental Disorders, Fifth Edition (DSM-5; American Psychiatric Association, 2013) among addictive substances. Unfortunately, this DSM-5 listing of benzodiazepines has been used by Jauhar et al. (2019) as an example in their argument against putative addiction to antidepressants.

We would like to address two main issues here. The first is the looseness with which the terms "addiction" and "addictive" are used. The second is the erroneous portrayal of benzodiazepine dependence and abuse in much of the literature and as exemplified by Jauhar et al. (2019).

\section{What is addiction and what makes a substance addictive?}

Jauhar et al. (2019) correctly note that withdrawal symptoms that occur upon discontinuation of medications prescribed for valid medical reasons do not suggest a substance-related disorder. However, they provide Leshner's (1997: 45) definition of addiction as "compulsive drug seeking and use" and contrast antidepressants with benzodiazepines by stating that benzodiazepine dependence is characterized by "compulsion. . . in those who abuse them." "Compulsion", like "dependence" and "abuse" is not well defined by Jauhar et al. (2019). This entire discussion also correctly implies that antidepressant dependence itself does not exist, despite withdrawal symptoms upon discontinuation of these agents. If this implication is accurate, withdrawal symptoms that occur upon cessation of any substance, including benzodiazepines, should not be construed to reflect dependence on or abuse of that substance.

It is important to note that "addiction" is not a term clearly endorsed by the DSM-5 (American Psychiatric Association, 2013). Addiction does not appear in its glossary of technical terms and is not defined elsewhere in the DSM-5. The only mention of addiction is via the name of the nosological group of "substancerelated and addictive disorders", but the DSM-5 (American Psychiatric Association, 2013) does not define "addictive disorders." In the introductory description of substance-related and addictive disorders, the DSM-5 states that the substances in question "produce such an intense activation of the reward system that normal activities may be neglected" and that these "drugs of abuse directly activate the reward pathways" producing "feelings of pleasure" (p.481). It follows that all 10 classes of substances encompassed by this nosological group, including benzodiazepines, are effectively considered "drugs of abuse" and that they all produce a "high." However, plenty of evidence suggests that

${ }^{1}$ Department of Psychiatry, Wayne State University, Detroit, MI, USA 2Department of Behavioral Neurosciences, Wayne State University, Detroit, MI, USA

${ }^{3}$ Department of Anesthesiology, Wayne State University, Detroit, MI, USA ${ }^{4}$ Department of Psychiatry, Tufts University, Boston, MA, USA

${ }^{5}$ Sydney Medical School, University of Sydney, Sydney, NSW, Australia ${ }^{6}$ Department of Health Sciences, University of Florence, Firenze, Italy ${ }^{7}$ Department of Psychiatry and Neuropsychology, Maastricht University, Maastricht, The Netherlands

${ }^{8}$ Department of Psychiatry, Queens University, Kingston, ON, Canada

${ }^{9}$ Institute of Psychiatry, Federal University of Rio de Janeiro, Rio de Janeiro, Brazil

${ }^{10}$ University of Pennsylvania, Philadelphia, PA, USA

${ }^{11}$ Department of Immunology, Tufts University, Boston, MA, USA

*The contribution of the first three authors is equal and they are listed in alphabetical order.

Corresponding author:

Richard Balon, Department of Psychiatry, Tolan Park Building 3A, 3901 Chrysler Service Dr., Detroit, MI 48201, USA.

Email: rbalon@wayne.edu 
when it comes to benzodiazepines, this is not true. Unfortunately, the mere fact that benzodiazepines are listed among the DSM-5 substance-related and addictive disorders is often interpreted as an official endorsement of the position that benzodiazepines are "addictive."

Interestingly, the DSM-5 (American Psychiatric Association, 2013) does include a diagnostic category of "antidepressant discontinuation syndrome," which is placed in the nosological group of "medication-induced movement disorders and other adverse effects of medication." Besides avoiding the term "antidepressant withdrawal symptoms," the DSM-5 is also careful not to attribute these "discontinuation symptoms" to antidepressant dependence or to a hypothetical "antidepressant use disorder" and indeed, the term "antidepressant dependence" does not appear in the DSM-5 text. The DSM-5 also makes this puzzling assertion: "The antidepressant discontinuation syndrome is based solely on pharmacological factors and is not related to the reinforcing effects of an antidepressant" (p.713). Such an approach begs the question of the explanation for "antidepressant discontinuation syndrome:" what causes it, where does it come from, how does it differ from the symptoms of withdrawal from other substances, and are antidepressants really devoid of "reinforcing effects?" This is in contrast to the DSM-5 portrayal of the withdrawal symptoms associated with the cessation of benzodiazepines, where benzodiazepines are assumed to have "reinforcing effects." Moreover, although benzodiazepine withdrawal symptoms do not have to be present for making the DSM-5 diagnosis of sedative, hypnotic, or anxiolytic use disorder, their presence is often considered a manifestation of this disorder.

We also question whether "compulsion" should be the key criterion for making a substance addictive, an argument made by Jauhar et al. (2019) following Leshner's (1997) definition. Compulsivity is usually defined as a repetitive behavior (regardless of whether it pertains to using a substance or engaging in an activity) that is motivated by avoidance of the perceived negative consequences (e.g. withdrawal symptoms, distress, or feeling of anxiety) of ceasing the substance or activity. However, compulsivity is not sufficient for the definition of addiction because people who continue taking antidepressants or benzodiazepines mainly to avoid the anticipated withdrawal symptoms (or return of primary symptoms of their disorder) should not be considered addicted only on that basis. Other criteria for substance addiction include an urge or a craving that immediately precedes substance use, poor self-control over substance use, and continued substance use despite its adverse consequences (Potenza, 2006; Shaffer, 1999). Only the presence of all these criteria may warrant use of the term "addiction." In our opinion, the DSM should define addiction clearly in its future iterations if it continues to use the label "addictive disorders." The DSM should also stipulate the criteria that allow substances or behaviors to be considered addictive on the basis of this definition.

For now, we believe that when using the terms "addiction" or "compulsion in this context, it should always be stipulated what these terms encompass, i.e. how they are defined. Failure to do so opens the floodgates to a chaotic situation where arbitrariness reigns and where the presence of any component of addiction, or only the presence of withdrawal symptoms, is deemed sufficient to conclude that it is indicative of addiction or substance use disorder. Likewise, a disregard for the criteria for addiction would allow countless substances to be regarded as addictive - a dangerous situation with conceptually disastrous consequences for medicine.

\section{Erroneous portrayal of benzodiazepine dependence and abuse}

With regards to the issue of dependence, Jauhar et al. (2019) state that "Repeated studies have indicated that around 35\% of people will develop dependence, and these people appear more likely to had been taking benzodiazepines for longer periods ( $>5$ years) and have dependence-prone personalities (Murphy and Tyrer, 1991)" (pp.655-656). Although Jauhar et al. (2019) mention "repeated studies," they only cite the article by Murphy and Tyrer (1991). However, this article does not address the issue of dependence, though its title includes the term "benzodiazepine dependence." In fact, Murphy and Tyrer (1991) address benzodiazepine withdrawal and conclude somewhat differently that "withdrawal symptoms were greater in patients who had taken a benzodiazepine for $>5$ years and were most marked in those with personality disorders, predominantly dependent ones" (p.511). The putative definition of dependence by Murphy and Tyrer (1991, p.511) was "apparent withdrawal symptoms on reduction" (presumably, a reduction in the benzodiazepine dose). There were no other signs of dependence, addiction, or abuse in their patients. Patients in this study were actually people taking a prescribed benzodiazepine in regular dosage for six months or longer; unable to reduce or stop their drug because of apparent withdrawal symptoms; were taking no other psychotropic drugs; were taking their benzodiazepine in a daily dosage of 2-16 $\mathrm{mg}$ of diazepam or equivalent; were taking their drugs (sic) for anxiety or insomnia or related neurotic symptomatology; and wished to stop benzodiazepines and participate in this study. Thus, the presence of withdrawal symptoms was the only criterion of dependence which per se, as noted and acknowledged above, is not enough to establish a diagnosis of substance use disorder and may not be enough to denote the kind of dependence that is associated with actual substance abuse. In addition, the DSM-5 (American Psychiatric Association, 2013, p.551) notes that the criterion of withdrawal is not considered to be met for individuals taking sedatives, hypnotics, or anxiolytics (including benzodiazepines) under medical supervision. Furthermore, Murphy and Tyrer (1991) wrote about dependent or passive-dependent personality disorder, not about dependence-prone personalities. This distinction is important because the latter term might erroneously suggest that some people are inherently more likely to become dependent on certain substances.

Why have benzodiazepines been mentioned in this and in similar arguments by others? Because benzodiazepines are perfectly set up to become victims of muddled thinking about addiction: they produce prompt relief, they are prescribed for patients who are anxious about control, they have a withdrawal syndrome, they are commonly abused by polysubstance abusers, and they have had nobody to stand up for them since "Big Pharma" abandoned them in favor of antidepressants. If not for assuming a conclusion about benzodiazepines, one could just as easily present an analogy between benzodiazepines and antidepressants as being reassuring about their abuse potential, rather than suggesting possible abuse of benzodiazepines. 
The most informative question to ask about a drug's potential for abuse is not whether anyone abuses it, but whether those with no substance abuse history are likely to do so. People in the throes of addiction may find ways to abuse a great variety of substances, including antidepressants, anticonvulsants, benzodiazepines, and atypical neuroleptics. For instance, quetiapine is known by street names such as "Susie Q" or "baby heroin," gabapentin by "gabbies" or "johnnies," and both are recognized to have street value (Buttram et al., 2017; Theremissine, 2008); gabapentin has been added to the list of controlled substances in some US states. These substances are alike in that they produce fairly prompt subjective soothing effects, but little else. The way to gauge the risk of the true addictive potential of a drug is to measure the degree to which it reinforces its own administration or induces subjective euphoria. It is, in fact, difficult to induce animals to self-administer benzodiazepines (Ator and Griffiths, 1987), and people with no substance abuse history have been found unable to distinguish these medications from placebo (e.g. de Wit et al., 1986; Johansen and Uhlenhuth, 1980). Further, there is no scientific evidence that legitimate prescription of benzodiazepines to non-substance abusers leads either to their abuse or to the abuse of other substances.

The endless and seemingly unresolved debate about abuse, dependence, and addiction of various medications, especially benzodiazepines, is frequently ideological and stigmatizing, forgetting patients and their illness. It is clear that just because a patient has symptoms of withdrawal and anxiety after benzodiazepine is stopped does not constitute a reason to diagnose abuse, addiction, or dependence. As noted by Greenblatt et al. (1983) almost four decades ago, "Since benzodiazepines cure neither anxiety nor insomnia, symptom recurrence can be anticipated after discontinuation of the drug" (p.357). Anxiety and insomnia are chronic disorders which do not get cured by a period of treatment with benzodiazepines or antidepressants. Long-term treatment should always be considered. In their systematic review and meta-analysis of the risk of relapse after discontinuation of long-term treatment with antidepressants, Batelaan and colleagues (2017) pointed out that such discontinuation increased the odds of relapse compared with continuation of antidepressant treatment. Summary relapse prevalence rates for continuation treatment were $36.4 \%$ (30.8-42.1\%, $n=28$ studies) for the placebo group and $16.4 \%$ (12.6-21.1\%, $n=28$ studies) for the antidepressant group. There is also evidence of lower relapse rates in panic disorder patients treated with a benzodiazepine (clonazepam) (34.1\% after one-year follow-up) compared to those in patients treated with a serotonin reuptake inhibitor (paroxetine) $(61.8 \%$ after one-year follow-up) (Freire et al., 2017). These findings raise the old question: why do we label as dependent only patients who become anxious after cessation of treatment with benzodiazepines and not patients with the same outcome after cessation of treatment with antidepressants? In relation to benzodiazepines, Marjot (2012) asked how we can know whether such anxiety is symptom recurrence, symptom emergence (new symptom emerging once the dose was reduced or stopped), or symptom misattribution (i.e. patients blaming benzodiazepines for their current distress). In this context, Marjot (2012) warned of the post hoc ergo propter hoc ("after this, therefore because of this") logical fallacy, whereby it is assumed that dependence precedes and therefore causes withdrawal symptoms. Finally, if we consider the study by Batelaan et al. (2017) and its recommendation of chronic treatment of anxiety disorders, why should such treatment be justified only with antidepressants (which have their own issues) and not with benzodiazepines, when they are both associated with withdrawal symptoms upon their cessation?

\section{Conclusion}

In summary, we agree with Jauhar et al. (2019) that antidepressants are not addictive. This is based on the concept of addiction that does not include withdrawal symptoms, but encompasses craving for a substance, poor self-control over substance use, continued substance use despite its adverse consequences, and compulsive substance use. Espousing the consistency, while using the same criteria for addiction, we also emphasize that there are no reasons to consider benzodiazepines addictive. What is sorely needed is better understanding of the withdrawal symptoms, and of the pharmacological dependence that is often assumed to cause these symptoms, while resisting attempts to equate them with addiction.

\section{Declaration of conflicting interests}

The author(s) declared no potential conflicts of interest with respect to the research, authorship, and/or publication of this article.

\section{Funding}

The author(s) received no financial support for the research, authorship, and/or publication of this article.

\section{ORCID iDs}

Richard Balon (iD https://orcid.org/0000-0001-6598-2242

Vladan Starcevic (iD https://orcid.org/0000-0002-6772-6995

Rafael C Freire iD https://orcid.org/0000-0003-3875-4601

Antonio E Nardi (iD https://orcid.org/0000-0002-2152-4669

\section{References}

American Psychiatric Association (2013) Diagnostic and Statistical Manual of Mental Disorders (DSM-5 R). 5th ed. Arlington, VA: American Psychiatric Association.

Ator NA and Griffiths RR (1987) Self-administration of barbiturates and benzodiazepines: A review. Pharmacol Biochem Behav 27: 391-398.

Batelaan NM, Bosman RC, Mintingh A, et al. (2017) Risk of relapse after antidepressant discontinuation in anxiety disorders, obsessivecompulsive disorder, and post- traumatic stress disorders: Systematic review and meta-analysis of relapse prevention trials. BMJ 358: j3927.

Buttram M, Kurtz S, Dart R, et al. (2017) Law enforcement-derived data on gabapentin diversion and misuse 2002-2015: Diversion rates and qualitative research findings. Pharmacoepidemiol Drug Safety 26: $1083-1086$

de Wit H, Uhlenhuth EH, Hedeker D, et al. (1986) Lack of preference for diazepam in anxious volunteers. Arch Gen Psychiatry 43: 533-541.

Freire RC, Amrein R, Mochcovitch MD, et al. (2017) A 6-year posttreatment follow-up of panic disorder patients: Treatment with clonazepam predicts lower recurrence than treatment with paroxetine. $J$ Clin Psychopharmacol 37: 429-434. 
Greenblatt DJ, Shader RI and Abernethy DR (1983) Current status of benzodiazepines (First of two parts). N Engl J Med 309: 354-358.

Jauhar S, Hayes J, Goodwin GN, et al. (2019) Antidepressants, withdrawal, and addiction; where are we now? J Psychopharmacol 33: 655-659.

Johanson CE and Uhlenhuth EH (1980) Drug preference and mood in humans: Diazepam. Psychopharmacology (Berlin) 71: 269-273.

Leshner AI (1997) Addiction is a brain disease, and it matters. Science 278: $45-47$.

Marjot S (2012) Comment on Lader (2011). Addiction 107: 684.
Murphy SM and Tyrer P (1991) A double-blind comparison of the effects of gradual withdrawal of lorazepam, diazepam and bromazepam in benzodiazepine dependence. Br J Psychiatry 158: 511-516.

Potenza MN (2006) Should addictive disorders include non-substancerelated conditions? Addiction 101(Suppl 1): 142-151.

Shaffer HJ (1999) Strange bedfellows: A critical view of pathological gambling and addiction. Addiction 94: 1445-1448.

Theremissine OV (2008) Is quetiapine a dug of abuse? Re-examining the issue of addiction. Expert Opin Drug Saf 7: 739-748. 